\title{
Interrelationship Superstructure for Mobile Communications
}

\author{
Maninder Kaur, Arthi Srivastava, Abhishek Ganu \\ (ECE/Keshav Memorial Institute of Technology/JNTU/India) \\ (CSE/KMIT/JNTU/India) \\ (CSE/KMIT /JNTU/India)
}

\begin{abstract}
This article describes suitable network architecture for the mobile communications system beyond IMT-2000. Recent rapid developments in mobile communication services have significantly altered the lifestyles of many people. With the increasing demand for access to the Internet, the amount of data traffic in mobile communication networks has significantly increased and will dominate these networks. Therefore, mobile networks beyond IMT-2000 should offer higher bit rate channels and adapt to multimedia traffic more efficiently. This article first forecasts market trends in mobile communications in order to identify requirements of the mobile communications system beyond IMT-2000. Then the article considers the network architecture of the mobile communications system beyond IMT-2000 that will satisfy those requirements.
\end{abstract}

Keywords: Architecture, IMT 2000, Multimedia traffic, Network architecture

\section{Introduction}

The concept of "anytime, anywhere, anyone" was realized by the development of mobile communications systems for voice communications. Initially, mobile communication systems were mainly used by a few people as a complement to fixed telephone communications., the number of subscribers has significantly increased, driven by the continuous development of mobile technologies, the expansion of service areas, the introduction of compact terminals, and the increased popularity of mobile communications among the younger generation. Data communication services through mobile networks have attracted many people to the WWW and have promoted the exchange of e-mail. These services are now expected to change people's lifestyles dramatically through further development of mobile communication technologies. Thus, the status of services of mobile communication systems is changing from "complementary" to "requisite" and their role is expanding from "handy phone" to "private information infrastructure."

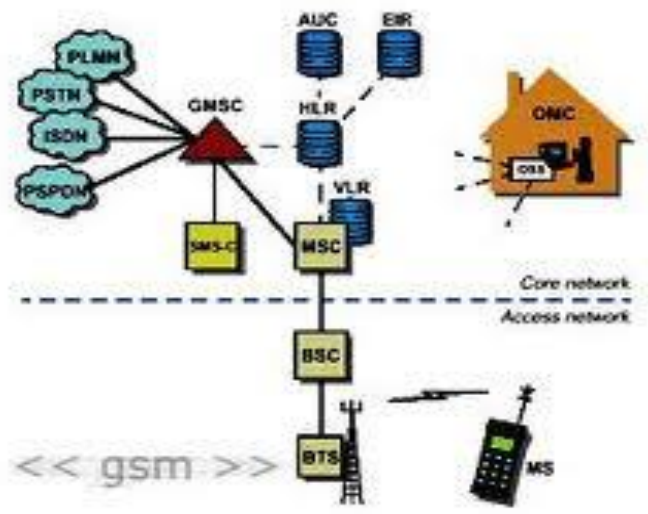

Figure 1. Basic Network

The third-generation (3G) system known as the IMT-2000, which will be introduced in 2001, will enhance the ability of data communications. The system offers channels of less than $2 \mathrm{Mb} / \mathrm{s}$ when subscribers move at vehicular speeds. Therefore, the mobile communications system beyond IMT-2000 (4G) should be designed to offer significantly higher bit rates than $2 \mathrm{Mb} / \mathrm{s}$ even in a vehicular environment and to adapt to data communications more efficiently to realize the concept of "anytime, anywhere, anyone, and anything" from the viewpoint of multimedia communications.

This article first forecasts market trends in mobile communications in order to identify requirements of the mobile communications

beyond IMT-2000. The article proposes a 4G-systemn network architecture that satisfies those requirements. 
Interrelationship Superstructure for Mobile Communications

\section{Market Trends of Mobile Communications 4G System Objectives}

Taking a retrospective view of the history of mobile communications, we can see that a technological revolution in mobile communications or a major replacement in facilities has occurred every decade. Figure 1 shows the generations ofmobile systems and their application areas from the viewpoints of transmission bit rate and the mobility of terminals. As the figure shows, the milestones achieved in mobile communications aim at faster bit rates and wider service areas. The first-generation system, which was deployed in the 1980s, was based on analog FM transmission technologies; the second- generation (2G) system applied digital transmission tech nologies such as TDMA. Both generations are mainly used for voice communications, although the $2 \mathrm{G}$ system offers data communication services using digital channels at several tens of kilobits per second

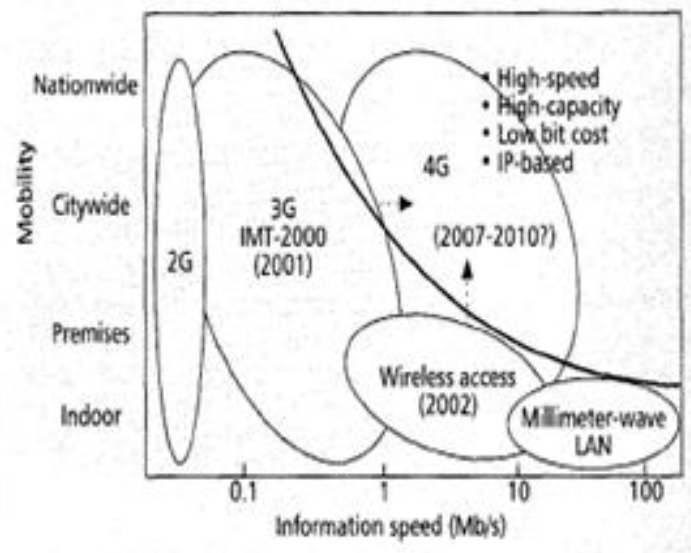

Figure 2. Information speed

The $3 \mathrm{G}$ system achieves a maximum bit rate of $2 \mathrm{iMb} / \mathrm{s}$ and will offer packet- switched multimedia services as well as circuit switched voice services. Because the $4 \mathrm{G}$ system will most likely be introduced several years thereafter, a bit rate higher than that of the $3 \mathrm{G}$ system should be offered even in a high-speed mobility environment. From the aspect of other than the transmission bit rate, the $4 \mathrm{G}$ system will be expected to connect not only pe0p.e but also machines and people. In the 21 st century our society will be more information-oriented and all information will be digitized. Most electrical appliances will be equipped with communication functions. The ratio of data traffic to voice traffic will significantly increase. In addition, through enhanced mobile terminals such as PDAs (personal digital assistants), people will be able to access nesccesary information and ccintrol their appliances remotely whenever and from wherever they wish. In order to realize such a society, the $4 \mathrm{G}$ system should be designed to seamlessly connect

people and their environment.

\section{Traffic Estimation}

If mobile communicaticns are used only for voice communications between people, the number of subscribers and traffic volume will become saturated in the near future. However, mobile communications will provide data communications between non-human objects as well as people. Therefore, the amount of mobile comrnunications traffic will further increase for a considerable time into the future based on the development of new applications.

In February 1999 " i-mode service" was introduced in Japan. This service covers a wide range of applications, such as enabling customers 10 access Web sites, exchange mail, and buy tickets . Since the service was introduced the number of i-mode subscribers has dramatically increased. At the end of March 2001 the number of mobile Internet subscribers was 3.5 million in Japan (a total of three operator groups). Furthermore, there are other factors that will increase the amount of mobile traffic in the future. For example, one user may use several mobile terminals (MTs) depending on a given situation. Many object:; equipped with communication functions such as multimedia vehicles, electrical appliances, vending machines, and pet locators, will proliferate and diversify the demand for mobile communications. Table 1 gives the statistics of population, vehicles, and personal computers in some countries to show such potential demand. Considering these market trends, mobile communications traffic in 2010 and 2015 is estimated as shown in Fig. 2. This estimation is based on the ITU-R report M.2023, "Spectrum requirements for IMT-2000," by ITU-R Task Group 8/1. The TG8/1 estimation classified services that will be available in 2010 into six categories according to the channel bit rate: Speech (16 kb/s in each direction) Simple message (14 kb/s in each'direction) Switched data (64 kbi's in each direction) Medium multimedia (downlinlduplink 384/64 kb/s) High multimedia (do.wnlink/uplink: 2000/128 kb/s) Highly interactive mLltimedia $(128 \mathrm{~kb} / \mathrm{s}$ in each direction) The amount of 
traffic was estimated by assuming the number of subscribers and frequency of use of each service catego ry. "High multimedia" service, $2-\mathrm{Mb} / \mathrm{s}$ downlink and $128 \mathrm{-kb} / \mathrm{s}$ uplink, is the fastest service of the six categories. The amount of traffic of voice services that are representative services in the $1 \mathrm{G}$ and $2 \mathrm{G}$ systems is estimated to increase twofold in 2010 compared with that in 1999.

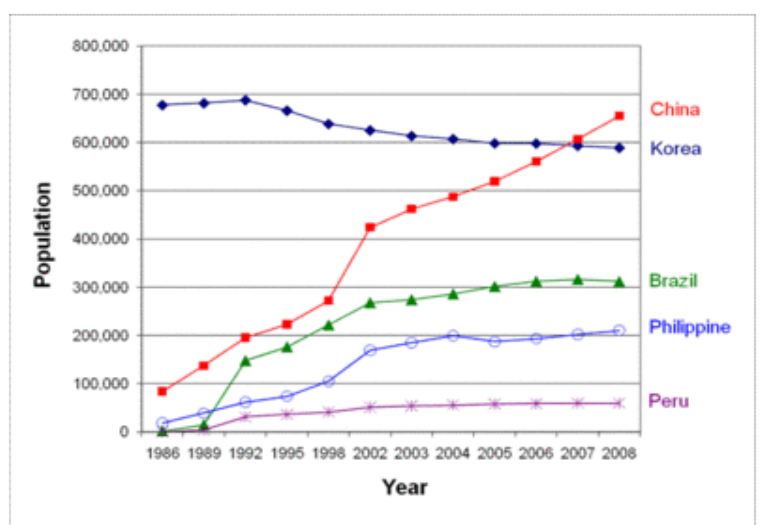

Figure 3. No. of subscribers according to population

Multimedia services will expand beyond those of voice in the $3 \mathrm{G}$ era and multimedia traffic will become twice that of voice services in 2010. To estimate the traffic in 201.5, we assume here that voice-service traffic will be saturated after 2010, multimedia traffic will grow at a 40 percent rate per year after 2010, and higher bit rate multimedia services (indicated as multimedia will be introduced. The 40 percent increase rate is based on two factors:

The capacity of memory and hard disks has increased at the The number of pixels in a CCD for input devices has also Based on these assumptions, the amount of traffic in 2015 will be 23 fold that of the present, and multimedia traffic will account for 90 percent of the traffic. As indicated in this estimation, 4G systems should accommodate this dramatically

increasing amount of multimedia traffic. Therefore, enhancing the system capacity as well as achieving a higher bit rate transmission are important requirements for the $4 \mathrm{G}$ system.

\section{Network Requirements}

In this section, possible wireless transmission characteristics for the $4 \mathrm{G}$ system are described. Then requirements for the network are discussed considering these characteristics. Wireless Transmission Charateristics Transmission Bit Rate - The 3G system achieves a maximum bit rate of $2 \mathrm{Mb} / \mathrm{s}$, but the bit rate may decrease in a vehicular- speed environment. Wireless LANs and other broadband wireless access systems using 5-GHz frequency bands (e.g., MMAC , IEEE 802.11, and HiperLAN/2 will offer greater than 30-Mb/s transmission capability in an indoor/pedestrian environment. For the $4 \mathrm{G}$ system, more than $20-\mathrm{Mb} / \mathrm{s}$ transmission will be realized in an outdoor/vehicular environment Spectrum - Because the $4 \mathrm{G}$ system will provide greater than $20-\mathrm{Mb} / \mathrm{s}$ wireless channels and should accommodate the significantly increasing amount of traffic, sufficient frequency

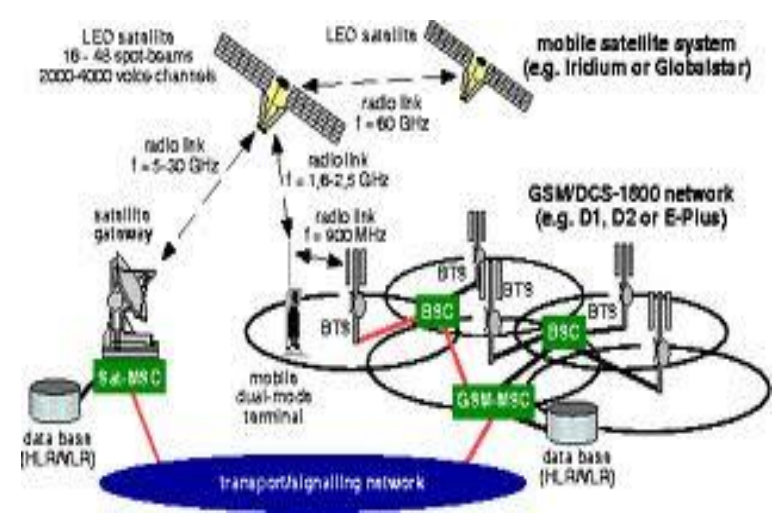

Figure 5. Different types of Mobile switching center 


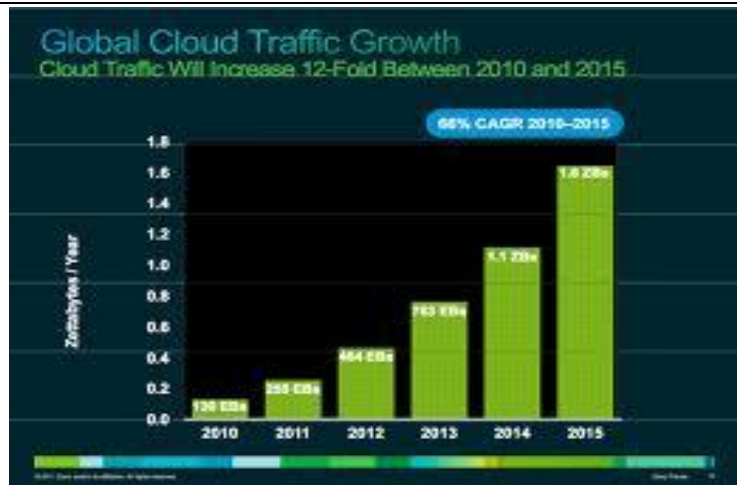

Figure 5. Traffic Growth

resources will be required. A lower frequency band, which is considered suitable for mobile communications, is now heavily used. Therefore it seems unlikely that a frequency band below $3 \mathrm{GHz}$ will be used for the $4 \mathrm{G}$ system. Although the frequency band for the $4 \mathrm{G}$ system has not yet been discussed in the ITU-R (WRC), the 4G system design should consider a higher frequency band for operation.

\section{Area Coverage}

One of the advantages of mobile communications is the ability to communicate independently of time and place. Therefore, expanding the service area of mobile communication systems is always an important issue for system operators. The $2 \mathrm{G}$ system now covers approximately 100 percent of populated areas, and customers can use mobile phones even in some buildings and underground shopping malls in urban areas. The 4G system is expected to have coverage similar to the $2 \mathrm{G}$ system. The $4 \mathrm{G}$ system will offer channels of more than $20 \mathrm{Mb} / \mathrm{s}$, which is three orders of magnitude greater than that of the $2 \mathrm{G}$ system.

The cell radius covered by a base station (BS) generally decreases if, assuming all other conditions are the same, radio signals are transmitted at higher bit rates because the received signal level must be higher than that at a lower transmission bit rate to compensate for the increased noise level. Moreover, as mentioned above, the $4 \mathrm{G}$ system may be operated at a higher frequency band so that propagation loss of the wireless signal is higher than that of $2 \mathrm{G}$ and $3 \mathrm{G}$ systems.

\section{Hierarchical Service Area}

Although we expect that all objects will be connected to a network through wireless links, it may be difficult for small devices to be directly connected to the $4 \mathrm{G}$ system due to power consumption and antenna size.

However, compact devices will be capable of exchanging wire-

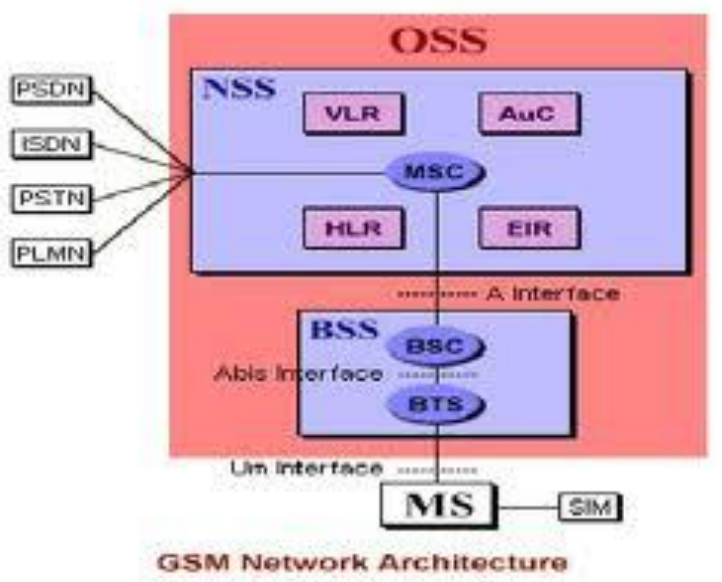

Figure 6. GSM Architecture

less signals at short range. Therefore, compact devices will be able to access the $4 \mathrm{G}$ nctwork through a miniature BS, which will act as a MT for the $4 \mathrm{G}$ system. By employing such a configuration, service areas will consist of multiple overlapping cells. 


\section{Requirements}

Seamless Connections - Imagining the network services in 2010, many types of wireless communication systems will be more popular as well as wired communications systems, and they will be used according to consumer needs The $3 \mathrm{G}$ system will play a major role in public mobile communication services, and wireless LANs will play a major role in private area communications. Short-range wireless systems will also be used to configure pi:rsonal area networks (PANs). These PANs are very short-rang networks established around a person in which very closely dispersed personal devices for information or communication such as personal computers, PDAs, and mobile terminals exchange various types of information In addition to these wireless access systems, the $4 \mathrm{G}$ system will offer several tens of megabits per second channels for public mobile communications. When many types of networks can be used, customers may wish to access each system according to time, location, or other conditions. Concerns pertaining to the economic coverage of the $4 \mathrm{G}$ system of rural areas can be eased by The complementary use of other wireless systems. For these purposes, the following functions will be required:

Interconnection between wireless access networks.

Capability of handover between wireless access networks.

Security mechanisms across wireless access networks.

\section{Reduction in the Nuniber of Control Messages and Short Delay at Handover}

As mentioned in the previous section, it seems unavoidable that the cell radius of a $4 \mathrm{G}$ BS will decrease. Due to this, cell sojourn time of customers will be shortened and the handover frequency will increase. Since channel bit rates of th: $4 \mathrm{G}$ will be enhanced beyond that of the $3 \mathrm{G}$ system, the number of error bits of packets will increase in the $4 \mathrm{G}$ system even with the same duration of channel cutoff. Therefore, reducing the number of control messages and a short delay handover are required.

\section{i) Reduction of Bit Cost}

Because customers have a limit as to how much they are w'illing to pay for services, the cost for communication services will not be significantly increased.

Therefore, to develop broadband multimedia communications, the bit cost of the 4G system should be decreased as well as the realization of broadband transmission capability. As mentioned above, the cell radius of the 4G system will tend to decrease based on traffic and propagation characteristics. If the decreased-area cells are used to cover entire service areas even in rural areas, the cost of the 4G system may approach an unacceptable level. In order to prevent this, the $4 \mathrm{G}$ network should be designed to reduce system costs while covering the largest possible service area.

\section{ii) Service integration Based on Internet Protocols}

Nowadays, most telecommunication services tend to be realized through the Internet and terminals tend to be connected to the Internet. In addition, many appliances will be networked and they will communicate with each other through the Internet [lo]. Therefore, the $4 \mathrm{G}$ network should support IP protocols as a future network infrastructure.

\section{iii) Movable Network Support}

Because the $4 \mathrm{G}$ system will offer sufficiently high-speed channels, a LAN in a vehiclewill be connected as a movable network to the $4 \mathrm{G}$ system. MTs may be connected to such movable networks and may communicate with the $4 \mathrm{G}$ system through the movable network, or compact devices connected to a PAN will be connected to the $4 \mathrm{G}$ system through a MT in the PAN. In this way, movable networks or MTs in the $4 \mathrm{G}$ system will work as a gateway for smaller networks. Therefore, the $4 \mathrm{G}$ system should consider accommodating a movable network as well as the traditional MTs

\section{Basic Concept}

\section{Network Architecture}

Similar to the 3G system, it comprises a core network (CN), which performs location control, call control, and service control; and the radio access network (RAN), which performs radio transmission and radio resource control. Mobility control is performed through cooperation between the $\mathrm{CN}$ and RAN. The information transportation in the 4G system (CN and RAN) is based on IP protocols. Each MT has its own IP address. Therefore, the 4G network directly connects with the Internet, but connects with ISDN or PSTN through gateways. Contrarily, traditional mobile systems connect to the Internet through gateways. In order to make seamless connections, multiple types of RANs are connected to a common $\mathrm{CN}$. 


\section{i) RAN Physical link Configuration}

In order to realize economical high-speed large-capacity mobile communication systems, deployment of the RAN is a key issue to be resolved. The configuration of the RAN of the 3G system (3G-RAN) is shown in Fig. 5a. It has a vertical-tree structure and multiple BSs are connected to a radio network controller (RNC). In order

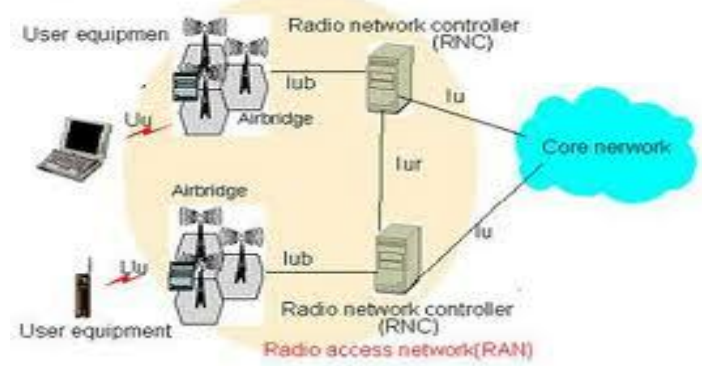

Figure 7. Terminals of the network

to enhance radio-signal quality at the cell edges, a diversity handover scheme is introduced to the $3 \mathrm{G}$ system. In the scheme, when a MT can communicate with multiple BSs, that is, the MT is handed over between adjacent BSs, all Layer 1 signals of the uplink (MT to BSs) received at each $\mathbf{B S}$ are transmitted to the RNC, and the RNC combines them into a user data stream. Simultaneously, Layer 1 signals of the downlink (BSs to MT) are transmitted from the multiple BSs, and the MT combines the received data from multiple BSs into a user data stream. If three BSs are incorporated for each MT handover, all Layer 1 signals on the BS approach links between the RNC and the BSs are tripled compared to the original user information. In the 4G system, which employs decreased-area cells and in which handover may occur more frequently, the load on both the approach links and the RNC signal processing equipment will be heavier. This will cause a serious cost increase in the RAN. Thus, a'new and innovative RAN structure should be studied to reduce the infrastructure cost. The RAN structure proposed here is a cluster type-RAN as shown in Fig. 5b. In the figure, BSs are grouped into a "cluster" and there is a "cluster-head" BS, which is connected to the CN. BSs in a cluster are linked to each other by a kind of LAN (horizontal structure) and diversity hand-over in the cluster is processed in a distributed manner within the cluster (localized handover processing). The cluster is viewed as a "virtual BS" from the core network. Thus, most of the Layer 1 signal processing is enclosed in the cluster so that the load on both the approach links and the signal processing on the specific equipment are significantly reduced. Figure $5 \mathrm{~b}$ also shows the Layer 1 signal processing concept. The uplink signal is received by multiple BSs and then the received signal is forwarded to one of the BSs that processes the uplink signal as a temporal agent. After the processing, the uplink signal is forwarded to the $\mathrm{CN}$ by the cluster-head BS. As the MT moves, the representative $\mathbf{B S}$ (the temporal agent) is changed to one of the adjacent BSs.

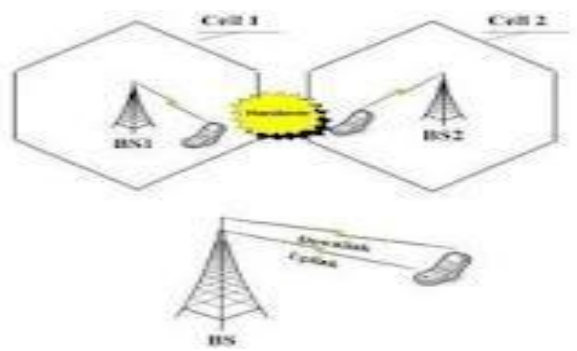

Figure 8. Handover

Downlink packet signals are multicast from the cluster-head BS to the other BSs in the cluster. When a MT can communicate with multiple BSs simultaneously, the downlink quality and required transmitting power for each MT can be monitored and fed back to the BSs with a short time delay. With this information, each BS autonomously determines whether or not it is better to transmit a packet signal. Thus, the probability of unnecessary or excessive-power transmission will be reduced. This realizes high-efficiency and low interference transmission so that the capacity of the $4 \mathrm{G}$ system will increase. 


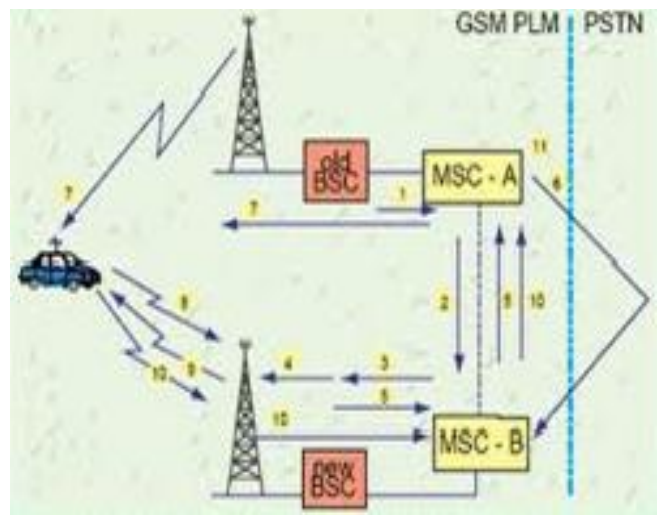

Figure 9. Mobile Station gets signal from Base Station

\section{Conclusion}

This article analyzed the market trends of mobile communications and identified the requirements for the mobile communications system beyond TMT-2000. The amount of traffic of future mobile communications is projected to increase significantly.

To accommodate this huge amount of traffic, the mobile communications systems beyond IMT-2000 will use a higher frequency band and offer information channels that have a bit rate ten-fold higher than the $3 \mathrm{G}$ system. Due to this, the cell radius of the mobile communications system beyond IMT-2000 will decrease and the handover frequency will increase. Moreover, since several types of radio access networks will be used in the future, seamless handover between these radio access networks will be necessary. Based on these requirements, this article presented network architecture for the mobile communications system beyond IMT- 2000 that comprises an IP-based core network and several types of radio access networks. According to this configuration, mobile communication systems are seamlessly connected to each other and the Internet. This article proposed a cluster- type link configuration, the $4 \mathrm{G}-\mathrm{RAN}$, to reduce air interface Layer-1 control signals and pointed out that the core network and the 4G-RAN are integrated into a unified IP transport network. The article summarized the mobility control. Other cellular network functions can also be developed in the IP transport network.

\section{References}

[1] M. Zeng, A. Annamalai, and V. Bhargava, "Recent Advances in Cellular Wireless Communications," /€E€ Commun. Mag., vol. 37, no. 9, Sept. 1999, pp. 128-38.

[2] K. Enoki, "Concept of i-Mode Service: New Communication Infrastructure in the 21st Century," NTT DoCoMo Tech. Journal, vol. 1, no. 1, Oct. 1999, pp. 4-9.

[3] ARIB, "MMAC," available at http://www.arib.or.jp/mmade/index.htmL

[4] ETSI, "HiperLAN/2 Standard," available at http://www.etsi.org/technicalactiv/hiperlan2. Html

[5] S. Abeta et al., "Coherent DS-CDMA and MC-CDMA Broadband Packet Wireless Access in a Multi-cell Environment," Proc. I€€€ VTC-2000 Fall, Sept. 2000, pp. 2213-18.

[6] F. Kojima, H. Harada, and M. Fujise, "Adaptive Sub-carriers Control Scheme for OFDM Cellular Systems," Proc. VTC2000 Spring, May 2000, pp. 1065-9.

[7] 1 1. Walfisch and H. L. Bertoni, "Theoretical Model of UHF Propagation in Urban Environments," I€€€ Trans. Ant. Prop., vol. 36, no. 12 , Dec.

[8] W. Mohr, "Broadband Radio Access for IP-Based Networks in the IST BRAIN Project," Proc. /E€€ ICT, May 2000.

[9] T. Siep et al., "Paving the Way for Personal Area Network Standards: An Overview of the IEEE P802.15 Working Group for Wireless Personal Area Networks," /€E€ Pers. Commun., vol. 7, no. 1, Feb. 2000, pp. 37-43.

[10] M. Nakagawa, "Wireless Home link," l€lC€ Trans. Commun., vol. €82-B, no. 12, Dec. 1999, pp. 1893-6.

[11] H. Soliman et al., "Internet-Draft, Hierarchical MIPv6 mobility management (draft-soliman-mobileip-hmipv6-02.txt)," Feb. 2001 